\title{
PREVALENCIA DE APIÑAMIENTO DENTARIO ANTERIOR Y SU RELACIÓN CON LA GINGIVITIS, EN ADOLESCENTES DE 12 A 15 AÑOS DE LA UNIDAD EDUCATIVA TRES DE NOVIEMBRE, PARROQUIA BELLAVISTA, CUENCA - 2015
}

\section{PREVALENCE OF ANTERIOR DENTITION CROWDING AND ITS RELATIONSHIP WITH GINGIVITIS, IN ADOLESCENTS AGED 12 TO 15 YEARS OF THE EDUCATIONAL UNIT TRES DE NOVIEMBRE, BELLAVISTA PARISH, CUENCA - 2015}

\author{
García-Soliz Valeria $^{1} *$, Zhunio-Ordóñez Karla ${ }^{2}$ \\ ${ }^{1}$ Odontóloga de práctica privada, Cuenca. Ecuador \\ 2 Odontóloga de práctica privada, Cuenca. Ecuador \\ *valerias1588@hotmail.com
}

\begin{abstract}
Resumen
OBJETIVO. Determinar la prevalencia de apiñamiento dentario anterior en dientes permanentes y su relación con la gingivitis, en adolescentes de 12 a 15 años de la Unidad Educativa Tres de Noviembre. MATERIALES Y MÉTODOS: El estudio fue de tipo descriptivo de corte transversal, el universo estuvo conformado por 214 escolares. RESULTADOS: Este estudio demostró que el $83 \%$ de los escolares presentaron apiñamiento dental, siendo más prevalente a los 12 y 13 años de edad, la gingivitis se presentó en un $37 \%$, con inflamación leve. CONCLUSIONES: Existe mayor apiñamiento dental inferior, lo que desencadena una gingivitis leve en ambos sexos.
\end{abstract}

Palabras clave: gingivitis, inflamación.

\section{Abstract}

OBJECTIVE: To determine the prevalence of anterior tooth crowding in permanent teeth and its relationship with gingivitis, in adolescents aged 12 to 15 years of the Educational Unit Tres de Noviembre. MATERIALS AND METHODS: The study was descriptive cross-sectional, the universe consisted of 214 schoolchildren. RESULTS: This study showed that $83 \%$ of schoolchildren presented dental crowding, being more prevalent at 12 and 13 years of age, gingivitis was presented in $37 \%$, with mild inflammation. CONCLUSIONS: There is greater inferior dental crowding, which triggers a slight gingivitis in both sexes.

Key words: gingivitis, inflammation.

\section{Introducción}

El apiñamiento dentario es un problema presente en los arcos dentales por ausencia de espacio para la ubicación de las piezas dentarias, donde intervienen factores genéticos, mal posicionamientos dentarios, etc; lo que obliga a tener una división de esta alteración, los factores que la favorecen y sus consecuencias. ${ }^{1,2}$ La gingivitis como consecuencia del apiñamiento dental se encuentra enfocada de acuerdo a la clasificación internacional existente y sus posibles causas en la presencia de ésta. ${ }^{3,4}$ Según datos de la Organización Panamericana de Salud (OPS) nueve de cada diez individuos alrededor del mundo está en riesgo de padecer cualquier tipo de patología bucal, que va desde caries hasta patologías de las encías (gingivitis, periodontitis). ${ }^{5}$ De acuerdo a la Organización Mundial de la Salud (OMS), la maloclusión dental ocupa la tercera posición de prevalencia dentro de las enfermedades en Salud bucal, luego de la caries dental y de las patologías periodontales. Según datos de la Organi- 
zación Panamericana de la Salud (OPS) en Latinoamérica, hay elevados niveles de incidencia y prevalencia de maloclusiones cuyos valores sobrepasan el $85 \%$ de la población. El apiñamiento se define como la anomalía más habitual, favoreciendo a la maloclusión cerca de $40 \%$ a $85 \%$, en este trabajo de investigación se obtuvieron porcentajes que se encuentran dentro de este rango lo que se deduce que el apiñamiento sigue siendo una de las alteraciones más comunes en nuestro medio y sin un cuidado adecuado en la limpieza bucal pueden desencadenar una serie de patologías, entre ella enfermedades gingivales tales como la gingivitis. ${ }^{6}$ Esta investigación aportará con una base de datos de todos los escolares que presenten apiñamiento dental y gingivitis para un futuro tratamiento. El objetivo del presente estudio fue determinar la prevalencia de apiñamiento dentario anterior en dientes permanentes y su relación con la gingivitis, en adolescentes de 12 a 15 años de la Unidad Educativa Tres de Noviembre, parroquia Bellavista, Cuenca - 2015. Para ello, se utilizó una regla milimetrada para obtener las medidas del ancho mesio-distal y ancho intercanino de los dientes, una ficha clínica en donde se registraron los datos obtenidos de cada uno de los escolares.

\section{MATERIALES Y MÉTODOS}

La investigación que se realizó es de tipo descriptivo de corte transversal, en una muestra de 214 escolares, divididos en 62 hombres que corresponde a un $29 \%$ y 152 mujeres con un $71 \%$ de la "Unidad Educativa Tres de Noviembre", los alumnos estudiados corresponden a las edades comprendidas entre los 12 a 15 años, con un porcentaje de $17,3 \%$ de 12 años, 26,6\% de 13 años, $29 \%$ de 14 años y con un $27,1 \%$ de 15 años de edad. Se obtuvo la aprobación del Comité Institucional de Ética de la Universidad Católica de CuencaEcuador, la autorización de la Directora de la Institución Educativa, el consentimiento informado de los padres de familia y el asentimiento del escolar. Los adolescentes de 12 a 15 años fueron entrevistados para la recolección de los datos necesarios para este estudio. Se evaluó clínicamente a los escolares; a cada estudiante se le pidió que abra su boca y con un pedazo de alambre de cobre se procedió a medir el ancho intercanino y esta medida fue trasladada a una regla, el mismo procedimiento se hizo en los incisivos y caninos superiores e inferiores, en los cuales con el compás de punta seca se midió el ancho mesio- distal de cada una de estas piezas, de igual manera se evaluó el índice gingival teniendo en cuenta el color, contorno, textura y hemorragia de las encías, los valores obtenidos se pasaron a la ficha de recolección de datos; posteriormente se procedió al análisis de los datos obtenidos.

\section{RESULTADOS}

Se encontró que 77 escolares no mostraron apiñamiento dental superior; 71 escolares presentaron apiñamiento dental leve; 58 escolares tuvieron apiñamiento dental moderado, mientras que 8 escolares exhibieron apiñamiento dental severo. (Tabla 1) De acuerdo a la edad los resultados del

\begin{tabular}{lcc}
\hline & FRECUENCIA & PORCENTAJE \\
\hline Ninguno & 77 & 36 \\
Leve & 71 & 33,2 \\
Moderado & 58 & 27,1 \\
Severo & 8 & 3,7 \\
TOTAL & 214 & 100 \\
\hline
\end{tabular}

Tabla 1. Apiñamiento dental superior.

apiñamiento dentario superior fueron de $38 \%$ a los 12 años, de $39 \%$ a los 13 años, de $37 \%$ a los 14 años y de $36 \%$ a los 15 años, es decir, que el apiñamiento tuvo un leve aumento con relación a la edad. (Gráfico 1).

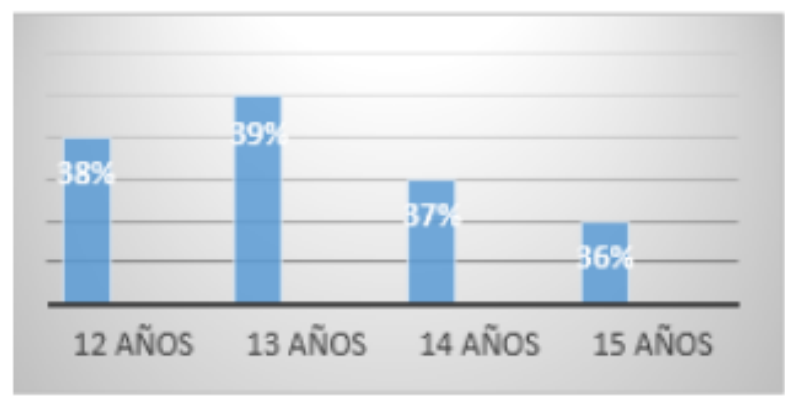

Grf. 1. Apiñamiento dental superior en relación a la edad.

Del total de estudiantes examinados se demostró que 178 escolares que corresponden a un $83 \%$ presentaron algún tipo de apiñamiento ya sea superior o inferior o combinados, mientras que 36 estudiantes es decir un $17 \%$ no presento apiñamiento. (Tabla 2).

En cuanto a la frecuencia del apiñamiento de la arcada inferior se observó que el $32,7 \%$ de escolares no presento apiñamiento dental, mientras que el $28,5 \%$ de escolares presentaron apiñamiento dental moderado. (Tabla 2)

El apiñamiento dentario en la arcada inferior mostró que a la edad de 12 años fue del $43 \%$, a la edad de 13 años fue de $35 \%$, a los 14 años fue de $31 \%$ y a los 15 años fue de $45 \%$. (Gráfico 3).

Al observar la frecuencia de Gingivitis los resultados fueron que 104 estudiantes no presentaron gingivitis $(48,6 \%)$; mientras que 80 escolares presentaron Inflamación gingival leve en un $37,4 \%$. (Tabla 3 ) 


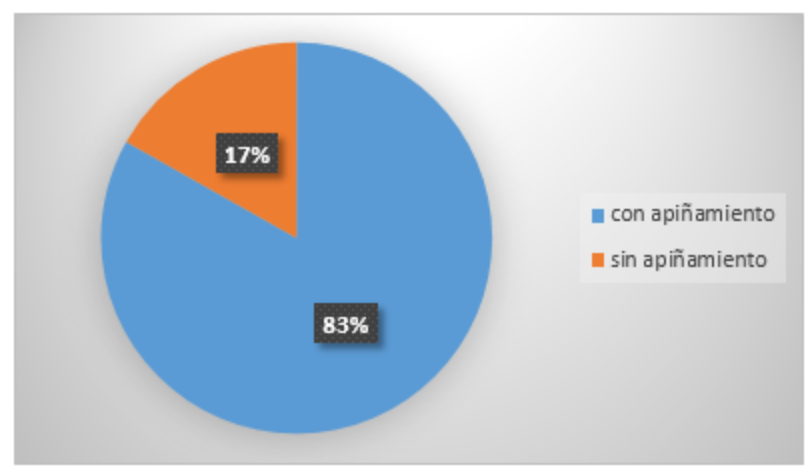

Grf. 2. Frecuencia de apiñamiento

\begin{tabular}{lcc}
\hline & FRECUENCIA & PORCENTAJE \\
\hline Ninguno & 70 & 32,7 \\
Leve & 80 & 37,4 \\
Moderado & 61 & 28,5 \\
Severo & 3 & 1,4 \\
TOTAL & 214 & 100 \\
\hline... &. &
\end{tabular}

Tabla 2. Apiñamiento dental en la arcada inferior.

\section{DISCUSIÓN}

Estudios realizados por Raza X, Pinto G y Ayala E, en el 2010, ${ }^{7,8}$ demostraron que de los 250 casos estudiados $56 \%$ de los pacientes presentaban apiñamiento. En nuestro estudio se puede apreciar resultados similares ya que se demostró que 36 escolares no presentaron apiñamiento, mientras que 178 escolares presentaron algún tipo de apiñamiento ya sea superior o inferior o combinados. Pérez, Navarrete en el 2010, ${ }^{9,}, 10$ concluyeron que el $62 \%$ de los estudiantes de 14 a 19 años de edad que fueron examinados en el colegio presentaron dientes desalineados. Por otra parte, en un estudio de prevalencia de apiñamiento dental en los estudiantes de la Facultad de Odontología de la Universidad Veracruzana $2011^{11,12}$ se obtuvo que el $58.8 \%$ de los estudiantes mostró algún tipo de apiñamiento dentario y el 41,2\% reveló ausencia de apiñamiento, lo que continua siendo una problemática que afecta a la mayor parte de la población. En cuanto a la frecuencia del apiñamiento dentario superior en nuestro estudio fue de $64 \%$ y el inferior de 67,3\%, comparando nuestros resultados con los estudios realizados en la Delegación Álvaro Obregón de la Ciudad de México ${ }^{13,14}$ concluyeron que el $58 \%$ de los escolares examinados presentaron apiñamiento dental, y de los niños estudiados el $20 \%$ presentó apiñamiento tanto en el maxilar superior como inferior. En otra investigación de

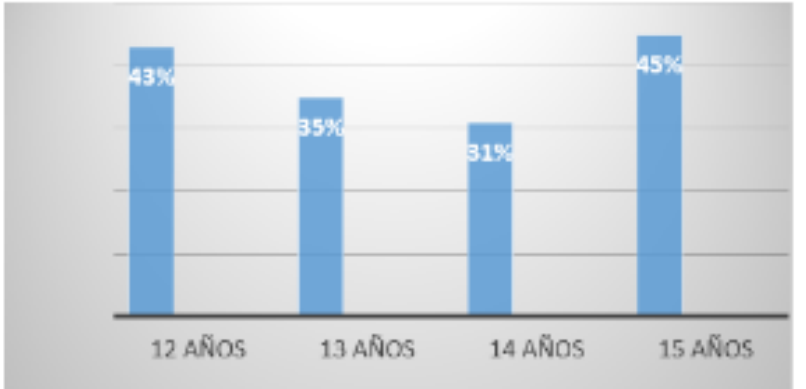

Grf. 3. Frecuencia de apiñamiento dentario inferior de acuerdo a la edad.

\begin{tabular}{ccc}
\hline & FRECUENCIA & PORCENTAJE \\
\hline $\begin{array}{c}\text { Encia } \\
\text { Normal } \\
\text { Inflamación } \\
\text { Leve }\end{array}$ & 104 & 48,6 \\
$\begin{array}{c}\text { Inflamación } \\
\text { Moderada }\end{array}$ & 24 & 37,4 \\
$\begin{array}{c}\text { Inflamación } \\
\text { Severa }\end{array}$ & 6 & 11,2 \\
TOTAL & 214 & 2,8 \\
\hline
\end{tabular}

Tabla 3. Frecuencia de gingivitis de la poblacion de estudio.

la aparición del apiñamiento dental con una muestra de 987 estudiantes del área de salud de Masó ${ }^{15,16}$ se comprobó que la frecuencia del apiñamiento dentario fue de 44,2\%; según el género, los hombres mostraron $49,2 \%$ y las mujeres, $50,8 \%$; el apiñamiento dentario en el maxilar inferior mostró 44,2\%; en el maxilar superior $23 \%$ y tanto en el maxilar superior como en el inferior el 32,8\%. La frecuencia del apiñamiento dentario se manifestó por encima del $40 \%$, presentando cierta similitud en cuanto al sexo, estos datos se relacionan a los datos obtenidos en nuestra investigación ya que existe un predominio de apiñamiento inferior tanto en hombres como en mujeres en un porcentaje de $37 \%$; además existe similitud en cuanto al género, en nuestro estudio se encontró que el apiñamiento dentario superior en el sexo femenino fue de $49 \%$, mientras que en los hombres el apiñamiento dental se presentó en un $51 \%$. Relacionando nuestros resultados con los datos obtenidos en el estudio en Portoviejo Manabí $2010^{17,18}$ en cuanto a la gingivitis inducida por acumulación de la placa bacteriana el $32 \%$ de los estudiantes examinados presentaron gingivitis y el $58 \%$ de los estudiantes no tenían gingivitis, estudios similares realizados en la Delegación 
Álvaro Obregón de la Ciudad de México, en correlación con el índice gingival el $60 \%$ de los escolares presentó cierto tipo de inflamación, prevaleciendo la inflamación leve, así mismo en nuestro estudio existió predominio de inflamación gingival leve en un $42 \%$. En un Proyecto Salud Bucal en Brasil en el año $2003^{19}$ en el municipio de Cáceres se realizó un estudio de las características de oclusión a 170 escolares, de ambos sexos, pertenecientes a 20 escuelas participantes de este proyecto en el cual se visualizó que la ocurrencia de la maloclusión fue del 78,82\%. Se llegó a la conclusión que la muestra investigada mostró un elevado índice de factores que componen la maloclusión y patologías colaterales por esta anomalía. Si relacionamos nuestros hallazgos con Pérez, Navarrete, ${ }^{20}$ en su trabajo muestra que el $62 \%$ de los estudiantes examinados presentaron apiñamiento dentario, en cuanto a la gingivitis el $32 \%$ de los escolares padecían de esta patología y el $58 \%$ de los estudiantes no tenían gingivitis, mientras que en nuestro estudio demostró que 178 escolares presentaban algún tipo de apiñamiento dental en un porcentaje de $83 \%$ y en cuanto a la gingivitis un $51 \%$ de escolares tenían inflamación gingival leve.

\section{CONCLUSIONES}

Se concluyó que 178 escolares presentaron algún tipo de apiñamiento ya sea superior o inferior o combinados en un porcentaje de $83 \%$, por lo que el apiñamiento sigue siendo un problema que afecta a la mayor parte de las personas. La frecuencia del apiñamiento dental en el maxilar superior fue de $64 \%$ y el inferior de $67,3 \%$. El apiñamiento dentario superior con respecto al género femenino se presentó en un $32 \%$, en el sexo masculino en un $33 \%$, siendo este último con mayor incidencia de apiñamiento dentario superior. El apiñamiento dental leve tanto superior como inferior se relacionó con una gingivitis leve en un porcentaje del $38 \%$. Los escolares que presentaron apiñamiento dental manifestaron cierta dificultad al momento de comer, hablar e incluso problemas bucodentales como consecuencia de la acumulación de placa, caries, enfermedad periodontal, además de repercutir en la estética del paciente, disminuyendo su auto estima.

Conflicto de intereses y financiamiento: Los autores declaran no tener conflicto de intereses, haber cumplido con los requisitos de autoría y haber autofinanciado este artículo.

\section{Referencias}

1 Organización Panamericana de Salud. La salud bucodental es esencial para la salud general, 2013 Marzo.

2 Castillo Preciado R. Prevalencia de apiñamiento dental en los alumnos de nuevo ingreso de la Facultad de Odontología de la Universidad Veracruzana. 2011; 32. (3, 35-65)

3 Balseca Troya, M. Estudio de la prevalencia de apiñamiento dentario anterior en la dentición permanente de adolescentes comprendidos entre 15-18 años de edad en el Colegio Nacional Ligdano Chávez de la ciudad de Quito. 2011.

4 Peréz Zipa M. Estudio del apiñamiento dental y su incidencia en las enfermedades gingivales de los estudiantes del Colegio “Teniente Hugo Ortiz” Portoviejo. 2010.

5 Quiros Alvarez O. Característica de la oclusión de los niños del jardín de Infancia Beatriz de Roche del Instituto Venezolano de Investigaciones Cientificas. IVIC. 2011.

6 Vélez Franco L. Pérdida de longuitud de arco como factor etiológico en el apiñamiento dental en los niños de 6 a 9 años de la escuela de educación básica Miguel de Cervantes Saavedra. 2014.

7 Carranza Fermín N, G. M. Periodontología Clínica. Editorial Mc Graw-Hill Interamericana. 2010. México 02 de febrero; 10 .

8 Raza X, Pinto G, Ayala E. Manual de educación para salud bucal para maestros y promotores. 2010.

9 Sánchez Perez L, Sáenz Martinez L, Alfaro Moctezuma P, Osorno Escareño C. Comportamiento del apiñamiento, gingivitis, higiene oral, caries, flujo salival y bacterias en escolares de 8 y 10 años. 2013.

10 Carmona Vidal E, Ayala Pérez Y, Díaz Morelli J. Apiñamiento dentario en escolares de 3 a 12 años. 2011.

11 Macías Gil R, Quesada Oliva L, Benítez Remón B, González García A. Frecuencia del apiñamiento dentario en adolescentes del área de salud Maso. 2009.

12 Méndez R. Tratamiento precoz del apiñamiento dental en denticion mixta: procedimientos de intervencion no habituales sin tocar dientes permanentes. Rev Esp Ortod. 2010.

13 Páramo A. Prevalencia de apiñamiento dental en niños con desnutrición de 6 a 9 años de edad en el municipio de Marques. Rev. Ortod. 2010.

14 Medina Seruto M, Gómez Mariño M, Quintero Ortiz J, Méndez Martínez M. Comportamiento de las enfermedades periodontales en adolescentes. AMC Camagüey. 2009; 13.(5.).

15 Vergara Villareal P, Cortes Uribe J, Ramirez Nubia R, Velasco Valdes E. Apiñamiento anteroinferior asociado a la presencia de terceros molares inferiores en la Ciudad de Cartagena. 2012. 
16 Matesanz Pérez P, Matos Cruz R, Bascones Martínez A. Enfermedades gingivales: una revisión de la literatura. 2008.

17 Arévalo C, Castro M, Losada S, Serrano M, Serrano C, Sabogal D. Guías de atención en Enfermedad gingival. 2012.

18 Ordoñez Vélez A. Apiñamiento dental. 2008.

19 Ruiz Bravo I. Análisis del ancho mesio distal coronario y discrepancias Dento - Dentarias en pacientes ortodónticos de la ciudad de Talca. 2008.

20 Orellana Manrique T. Alineación espontánea de los incisivos inferiores apiñados después de la extracción de los caninos temporales. 2008.

Recibido: 21 de enero de 2018.

Aceptado: 22 de febrero de 2018. 
